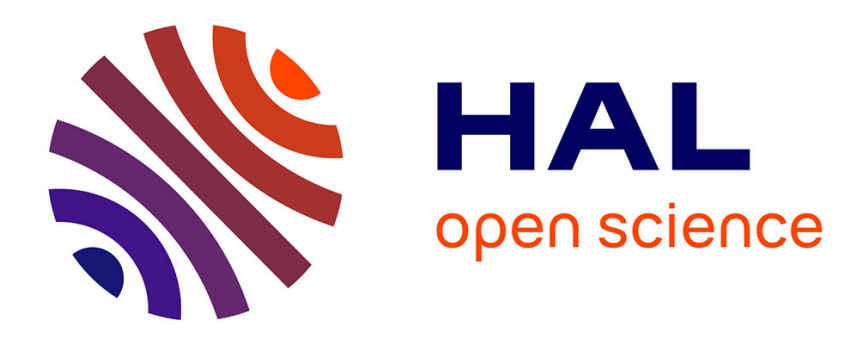

\title{
HIGH MAGNETIC FIELD SPECTROSCOPY IN ASTROPHYSICS
}

\author{
R. Garstang
}

\section{To cite this version:}

R. Garstang. HIGH MAGNETIC FIELD SPECTROSCOPY IN ASTROPHYSICS. Journal de Physique Colloques, 1982, 43 (C2), pp.C2-19-C2-28. 10.1051/jphyscol:1982203 . jpa-00221812

\section{HAL Id: jpa-00221812 https://hal.science/jpa-00221812}

Submitted on 1 Jan 1982

HAL is a multi-disciplinary open access archive for the deposit and dissemination of scientific research documents, whether they are published or not. The documents may come from teaching and research institutions in France or abroad, or from public or private research centers.
L'archive ouverte pluridisciplinaire HAL, est destinée au dépôt et à la diffusion de documents scientifiques de niveau recherche, publiés ou non, émanant des établissements d'enseignement et de recherche français ou étrangers, des laboratoires publics ou privés. 


\title{
HIGH MAGNETIC FIELD SPECTROSCOPY IN ASTROPHYSICS
}

\author{
R.H. Garstang \\ Joint Institute for Laboratory Astrophysios, University of Colorado and \\ National Bureau of Standards, and Department of Astro-Geophysics and \\ Department of Physics, University of Colorado,.Boulder, Colorado 80309, U.S.A.
}

Résumé.- La découverte de champs magnêtìques très intenses dans les êtoiles naines blanches et dans les étoiles à neutrons a fait naltre un grand intérêt pour la spectroscopie de $1^{\prime}$ atome placé dans de telles conditions. Nous rappelons 1 a découverte de la polarisation du spectre continu de certaines naines blanches et discutons 1 'interprétation qui confirme 1a présence de chanps magnétiques intenses. Des études récentes sur 1a classe d'étoiles doubles naines blanches magnétiques AM Herculis sont ensuite discutées ainsi que la découverte d'une raie spectrale discrète dans le pulsar du Crabe.

\begin{abstract}
The discovery of very large magnetic fields in white dwarf stars and neutron stars has stimulated interest in the spectroscopy of free atoms in high magnetic fields. We review the discovery of continuum polarization in certain white dwarf stars. We discuss the interpretation of the spectra of the magnetic white dwarfs and the confirmation of the presence of large fields. We describe recent investigations on a class of magnetic white dwarf binary stars (AM Herculis stars). Finally we mention the detection of a discrete spectrun line in the Crab pulsar.
\end{abstract}

1. Introduction.- Magnetic flelds are of much interest in astrophysics. This interest dates from 1908, when Hale discovered magnetic fields in sunspots from the Zeeman splitting of their spectral lines. The fields in large spots are a few thousand gauss. In the normal solar photosphere outside sunspots the fields are typically a few gauss. For many years there was no reason to expect that other kinds of stars would have magnetic fields much larger than those of the Sun. This situation changed when Babcock [1] demonstrated the presence of magnetic flelds in a number of stars of spectral type A, and In a few other stars. Many of these A stars show spectral peculiarities. Their fields are of order $10^{4}$ gauss. Many other types of stars have been examined for the presence of large magnetic fields, mostly without success. Success did come in 1970, however, and from an unexpected source. Magnetic white dwarf stars were discovered by Kemp, Swedlund, Landstreet and Angel [2] on the basis of circularly polarized continuum radiation and its subsequent interpretation in terms of a magnetic field of order $10^{7}$ gauss. Strong evidence has accumulated that pulsars have even larger magnetic fields, of order $10^{12}$ gauss. Finally a class of stars has been discovered, the prototype being AM Herculis, which are believed to be binary stars containfing a magnetic white dwarf star as one component and a companion star which is shedding matter from its outer layers on to the white dwarf. We shall review these discoverles, starting with single magnetic white dwarf stars.

2. Magnetic White Dwarf Stars.- Ordinary white dwarf stars have masses approximately the same as our Sun, luminosities which range from $10^{-2}$ to $10^{-4}$ of the solar luminosity, and surface temperatures in the range $4000^{\circ} \mathrm{K}$ to $80,000^{\circ} \mathrm{K}$. Their radii average 0.011 of the solar radius, slightly larger than the radius of the Earth. Their mean densities are $10^{6}$ times that of water. They show a variety of spectra. About 
two-thirds of the white dwarfs show hydrogen lines and are classified as DA type. Some show helium lines, a few show lines of neutral metals, and a signiffcant number show essentially continuous spectra with at most only very weak spectral features. White dwarfs are believed to be an end point of stellar evolution. They are composed of degenerate matter, except for a thin outer atmosphere. Several hundred white dwarfs are known: Greenstein [3] gave a compilation of 181 of them. A review on white dwarfs was given by Liebert [4]. The white dwarfs are believed to have evolved from normal stars. During this evolution conservation of magnetic flux would hold, and in a star whose radius decreases by a factor 100 the magnetic field strength would increase by a factor of $10^{4}$. For a typical main sequence star with a field of 1-10 gauss (if indeed most main sequence stars have fields comparable to that of the Sun, which is not known) the white dwarf evolutionary end-product would have a field in the range $10^{4}-10^{5}$ gauss, too small to be detected in white dwarf spectra at this time. Pulsar theories require fields of $10^{12}$ gauss, and if a pulsar has a radius of $10^{6} \mathrm{~cm}$ its progenttor must have had a field of $10^{2}$ gauss while it was on the main sequence. There are many pulsars, so many former main sequence stars must have had fields of this magnitude.

Many searches have been made for magnetic fields in white dwarfs. Preston [5] looked for quadratic Zeeman effect wavelength shifts in the Balmer lines of hydrogen, concluded that such shifts are absent in the DA stars he studied, and gave an upper limit of $5 \times 10^{5}$ gauss to the magnetic fields in the stars. Elias and Greenstein [6] set an upper limit of $10^{5}$ gauss for seven white dwarfs, and Angel and Landstreet [7] obtained a similar upper limit for nine white dwarfs on the basis of searches for polarization. other authors have made searches: the most extensive survey was by Ange1, Borra and Landstreet [8]. They observed over 100 white dwarfs. In selecting stars for observation they generally avoided the common DA and DB (helium) spectra, in which large magnetic fields could be detected by examination of the spectra for gross peculiarities, and concentrated on featureless and peculfar spectra. Thirteen stars in all show large flelds, over $5 \times 10^{6}$ gauss; these stars will be discussed below. The remainder of the stars show no measurable field. Perhaps the most interesting conclusion to a spectroscopist is that when a field is present it is strong enough to seriously distort atomic spectral features or even to obliterate them. There are no examples of more moderate fields which produce small linear Zeeman splittings or quadratic Zeeman shifts. In ten stars the upper limits on the field are 50,000 gauss, and for two stars (Wolf 1346 and 40 Eri B) the upper 1imfts are 10,000 gauss. The authors conclude that of about 350 known white dwarfs of type DA only five show large magnetic fields (the other eight magnetic white dwarfs are of types other than DA), so that perhaps 1 percent of white dwarfs may possess high magnetic fields.

We now turn to a discussion of individual magnetic white dwarf stars. Much additional information and more references to original papers may be found in the reviews of Garstang [9], Ange1 [10] and Ange1, Borra and Landstreet [8].

GD 90. Circular polarization was discovered by Angel, Carswel1, Strittmatter, Beaver and Harms [11]. They also discovered broad absorption features in the spectrum which were recognized to be resolved Paschen-Back structure in the Balmer lines $H \beta, H \gamma$ and $H \delta$. Greenstein [12] showed that $\mathrm{H} \alpha$ also has structure. By comparison with the calculations of Kemic [13] they deduced a field of $5 \times 10^{6}$ gauss . Polarization observations showed structure coinciding with the Paschen-Back components. Later work on this star includes [14].

BPM 25114. This star was discovered spectroscopically by Wickramasinghe and Besse1 [15]. The light varies with a period of 2.8 days. Subsequent investigations have Identified hydrogen lines with Zeeman splitting corresponding to a polar magnetic field strength of $3.6 \times 10^{7}$ gauss $[16,17]$.

L795-7 = Feige 7. This remarkable star was discovered by Liebert, Angel, Stockman, Spinrad and Beaver [18] to possess a large number of spectral features. These were identified as hydrogen and He I lines split into Zeeman components by a field of $1.8 \times 10^{7}$ gauss, determined by comparison of the spectrum with the calculations of 
Kemic [13]. The field is somewhat variable, with a period of 132 minutes and synchronously with variations in polarization. Presumably the star rotates, and an oblique rotator model is compatible with the observations. The narrowness of the spectral features indicates that the magnetic field must be rather uniform over a substantial part of the visible hemisphere of the star. Further observations of the star were obtained by Greenstein and Boksenberg [19], and by Greenstein and Oke [53] who obtained ultraviolet fluxes and some weak absorption features.

G35-26. D1scovered by Greenstein [20], this star shows Zeeman splitting of both H and He I In its spectrum, and there is a good match with the theory of Kemic [13] for a field of $8 \times 10^{6}$ gauss. The individual Zeeman components are remarkably sharp. The star has a high velocity and presumably belongs to population II. Polarization observations were made by Liebert and Stockman [45]; they failed to detect circular polarization, as might be expected for a low field.

699-47. This star shows an almost featureless spectrum $[12,21,22]$. However, Liebert, Angel and Landstreet [23] found a single feature at $6542 \AA$ which had a depth of 0.08 of the continum and was $30 \AA$ wide. Polarization observations [24] showed some evidence of structure in the 6000-7000 $\AA$ region. The discovery suggested that the feature is the central Paschen-Back component of $H \alpha$ shifted by 21 \& due to quadratic Zeeman effect, corresponding to a mean surface field of $1.6 \times 10^{7}$ gauss. The outer Paschen-Back components would be shifted and broadened out of visibility by the variability of the field over the surface of the star.

G99-37. Polarization was discovered by Landstreet and Angel [25] . The spectrum was found by Greenstein [26] to have unusual absorption features which have been attributed to $\mathrm{CH}$ and $\mathrm{C}_{2}$. A number of other spectral studies were made $[21,25,27]$, fo11owed by a detailed analysis by Angel and Landstreet [28]. They calculated individual CH line wavelengths as a function of magnetic field, and averaged over the molecular band structure. A fit of the profile of the band suggested a mean projected component of the magnetic field of $3.6 \times 10^{6}$ gauss.

IP 790-29. This strongly clrcularly polarized star shows an extremely strong absorption feature, stretching from 4400 to $5600 \AA$ (half-depth) and with a central depth of $75 \%$ of the continuum. Liebert, Ange1, Stockman and Beaver [29] suggest the feature is due to the $C_{2}$ swan bands in a field of $10^{8}$ gauss. This feature may well be the strongest absorption feature in any stellar spectrum.

Grw $+70^{\circ} 8247$. This star was the first magnetic white dwarf to be discovered, by Kemp, Swedlund, Landstreet and Angel [2]. They found broadband circular polarizat1on. Other polarization studies were made, including [30], [31], [32] and [33]. Some linear polarization is also present. The spectrum of the star is a longstanding puzzle. As long ago as 1938 Minkowski [34] discovered that the spectrum, previously considered featureless, had wide, shallow absorption features at $4135 \AA$ and $4475 \AA$. Greenstein [35] found another absorption feature at $3910 \AA$. The features are known as Minkowski bands. Greenstein and Matthews [36] confirmed these features and found others, as did Wegner [22,37] and Landstreet and Ange1 [33]. Numerous discussions of the origin of the bands have been made. The circular polarization establishes the presence of a strong magnetic field. Possible explanations of the features include quadratic Zeeman-shifted hydrogen Iines, neutral hellum, molecular helium and cyclotron emission. These suggestions have been reviewed $[9,10]$. Recent work includes new polarization observations (work of Angel, Stockman and Liebert reviewed in [10]) showing strong linear polarization at the wavelengths of the 4135 and $5855 \AA$ bands, indicating a field of more than $10^{8}$ gauss. In further discussion of the spectrum by Angel $[10,38]$ he points out that many Zeeman components of hydrogen lines show a stationary character at fields above $10^{8}$ gauss, so that the wavelengths do not vary greatly with increasing field. Angel suggests that the features in Grw $+70^{\circ} 8247$ are hydrogen 1 ine components at their approximately stationary wavelengths. If the wavelengths are nearly independent of field then there would be relatively little line broadening caused by the variation of field intensity and direction over the stellar surface. The features would not be broadened to the point of obliteration. Angel's suggested identification seems 
very likely to be the correct one, and if so a field of $2 \times 10^{8}$ gauss, or more, is indicated.

Greenstein and Oke [53] have made ultraviolet observations of the spectrum. The flux is far from being a smooth function of wavelength. There are large deviations from a blackbody and several relatively sharp features. The latter might possibly be due to $C$ I lines in a high magnetic field, and cyclotron absorption is also possible. But the absorptions must sti11 be considered as unidentified.

GD 229. This star shows both circular and linear polarization $[39,40,41]$. Its unusual spectrum shows a very strong absorption at $4185 \AA$ and a number of weaker absorptions $[12,19,22,42,43]$. U1traviolet spectrophotometry was obtained by Green and Liebert [44]. The absorption features could be due to cyclotron emission, or they could be due to a complex superposition of many Zeeman components of various elements. The ultraviolet data appear to rule out a large hydrogen abundance in the star, and a Zeeman interpretation of the features must be in terms of other elements, especially He $I$. In any case a field exceeding $10^{8}$ gauss would be required.

PG 1015+01. Discovered by Green (see review in [10]), this is a hot star with a rotational period of 1.6 hours. It has shallow but definite absorption features and shows variable circular polarization. The features have not been identified,

G195-19. Circular polarization was discovered by Angel and Landstreet [46]; it was the second polarized white dwarf to be discovered. Subsequent work on the polarization $[47,48]$ showed it was variable with a period of 1.33 days, an amplitude $0.24 \%$ and a mean $-0.23 \%$, indicating an oblique rotator. The spectrum is essentially continuous $[21,22,43,49]$. Perhaps all spectral features are broadened to invistbility by a high field.

G227-35. Circular polarization in this star ranges from $1 \%$ in the red to $3 \%$ in the violet and $8 \%$ in the infrared [50]. The spectrum is essentially continuous [12,50] with shallow depressions of the continuum, perhaps indicating that all spectral features are broadened to invisibility by a high magnetic field.

G240-72. This star shows strong linear and circular polarization [51]. The spectrum is essentially continuous, with very broad depressions of the continuum [12,22, 43,51]. Perhaps all spectral features are broadened to the point of invisibility by a high magnetic field of at least $10^{8}$ gauss. Cyclotron absorption is also a possible explanation of the features [52].

It is outside the scope of this lecture to discuss the origin and evolution of white dwarf magnetic fields and the effects of the fields on accretion phenomena. Interested readers are referred to the review by Angel [10]. The prevailing opinion is that the fields were frozen in at the time of formation of the white dwarfs, and there has not been time for the fields to decay by ohmic losses in the age of the Galaxy. The white dwarfs may have been formed from main sequence magnetic A-type stars; another possibility is that they were formed by the collapse of originally massive stars with carbon-burning cores, and convective amplification of the magnetic fields.

We mentioned above Angel's suggestion that the unidentified spectral features in $\mathrm{Grw}+70^{\circ} 8247$ are features of hydrogen and helium which are nearly stationary in wavelength as the field increases above $10^{8}$ gauss. Whether this same explanation applies to the unidentified features in GD 229 and PG $1015+01$ remains to be seen. Cyclotron emission seems an unlikely explanation: it would require too large a volume with a uniform field strength [19]. Further calculations of wavelengths of hydrogen and helium lines in fields of $10^{8}-10^{9}$ gauss will help to solve the problem.

3. AM Herculis Stars.- The variable star AM Herculis was discovered by Wolf in 1924 . Subsequent observations showed that it resembled the cataclysmic variables. Interest in the star became much greater following the suggestion by Berg and Duthie [54] 
that AM Herculis should be identified with a high galactic latitude X-ray source (known as $3 U 1809+50$ and as $4 \mathrm{U} 1814+50$ ). Hearn and Richardson [55] showed that the soft $X$-ray flux (0.1-0.4 keV) varles with a period of 3.1 hours, the same period which is shown by the optical 11ght curves of Szkody and Brownlee [56], the radial velocities of Cowley and Crampton [57] and Priedhorsky [58] and the polarization studies of Tapia [59]. This confirmed the identity of AM Herculis and the X-ray source. Subsequently a large number of additional observations have been made by many workers. We refer the reader to the excellent review article by Chiappetti, Tanzi and Treves [60] where an extensive list of observations and references may be found. We summarize a few of the leading characteristics of AM Herculis taken from this review article and from later papers. This will be followed by brief notes on some similat stars.

AM Herculis.

(a) The star shows a period of 0.128928 days, which is interpreted as the orbital period of a binary star.

(b) The light curve in the visual region shows a broad, deep primary minimum, a shallow secondary minimum, and much flickering. ('Primary minimum' is defined to be the deeper minimum in visual light.)

(c) In the Infrared both light minima are deep, the secondary minimum becoming increasingly deep at longer wavelengths and becoming the deeper minimum beyond 1.6 microns wavelength.

(d) In the blue and ultraviolet the secondary minimum is absent.

(e) There are long-term variations in the luminosity of the system. In the visual region the stellar magnitude varies from 12 (high state) to 15 (low state). The star spends most of its time in the high state.

(f) In the high state the spectrum shows strong emission lines of $\mathrm{H}$, He II, C IV, $\mathrm{N} V$ and other ions, superposed on a strong continuum. The continuum flux has approximately a $\lambda^{-2}$ wavelength dependence.

(g) The components of the binary are thought to rotate synchronousiy with the orbital revolution.

(h) The spectrum lines show radial velocity variations with the above period, but unlike normal eclipsing variables the maximum radial velocity of recession occurs at the time of primary minimum.

(1) In the high state the spectrum lines do not show any Zeeman splitting.

(j) Absorption 1ines of $\mathrm{Na} I$ at 8193-8194 $\AA$ are observed, presumably from the cool component of the binary system.

(k) The starlight shows both linear and circular polarization, varying with the orbital period. The linear polarization shows a sharp peak of $6 \%$, the circular polarization varies from $+3 \%$ to $-9 \%$, and the maximum linear polarization occurs at a phase when the circular polarization is zero. The polarization is very small in the near-ultraviolet.

(l) The X-ray flux vartes with the above period and has a minimum at the time of visible secondary minimum.

(m) The soft X-ray flux ( $<0.5 \mathrm{keV})$ is approximately fitted by a blackbody or by bremsstrahlung, with a temperature of $250,000^{\circ} \mathrm{K}$.

(n) Radio emission from AM Herculis was detected by Chanmugam and Dulk [61] w1th a flux density $6.7 \times 10^{-30}$ watts $\mathrm{m}^{-2} \mathrm{~Hz}^{-1}$ at a frequency of $4.9 \mathrm{GHz}$. There was no evidence of circular polarization. 
(o) Spectroscopy of the low state of AM Herculis has been accomplished by Schmidt, Stockman and Margon [62], Young, Schneider and Shectman [63], Latham, Llebert and Steiner [64] and Hutchings, Crampton and Cowley [65]. They find strong, sharp lines of $\mathrm{H}$ and $\mathrm{He} \mathrm{I}$. Absorption features close to the hydrogen lines are interpreted as Zeeman components in fields of $1.3 \times 10^{7}$ gauss. Tio bands are observed, as well as the Infrared $\mathrm{Na} I$ 1fnes. The continuum energy rises longward of $6500 \AA$. The star shows strong polarization $[62,64]$.

(p) Studies of the X-ray spectrum by Rothschild et al. [66] and by Tuohy, Mason, Garmi re and Lamb [67] covered from 0.1 to $150 \mathrm{keV}$. The hard X-rays ( $>2 \mathrm{keV}$ ) seem to be thermal bremsstrahlung.

There are many other details, which may be found in the review article and in the papers quoted. We proceed to discuss the interpretation of the observations.

The light curves and radial velocity curves clearly indicate that we are dealing with a binary system. One star is a very cool red dwarf, and the other is a small hot star, the hot star being eclipsed at primary minlmum. The strong polarization suggests that a high magnetic field is present. The detection of Zeeman splitting during the low state suggests that the lines seen are formed close to the hot star, and the hot star is probably a magnetic white dwarf star. The Na I lines and Tio bands probably arise from a dwarf star, whose spectral type has been estimated as M4.5 V or M5 V $[62,63,64]$. The mass of this star can be estimated in various ways, for example by assuming it is on the main sequence, or somewhat above 1t. A considered opinion [63] is that the mass is $0.26 \mathrm{M}_{0}$, the magnetic white dwarf star has a mass of $0.39 \mathrm{M}$ and a radius of $0.015 \mathrm{R}$. The distance of the system can be estimated from a spectroscopic parallax for the $M$ star, from a trigonometrical parallax, and from fitting of the observed continuous spectrum in the blue region to a standard DA type spectrum. The results obtained [62] are in the range 60-100 parsecs. Many of the features in the spectrum, particularly when the star is in the high state, do not arise from the stars themselves, but from streams of gas between the stars. The red dwarf probably fills its Roche lobe, and matter is spilling over and accreting onto the white dwarf star. The semimajor axis of the orbit is about $8 \times$ $10^{10} \mathrm{~cm}$. The radius of the red dwarf is about $3 \times 10^{10} \mathrm{~cm}$, that of the white dwarf (mentioned above) about $1 \times 10^{9} \mathrm{~cm}$. The radio observations can be interpreted as cyclotron emission by assuming a fleld strength of perhaps 50 gauss, and this might exist at about $8 \times 10^{10} \mathrm{~cm}$ from the white dwarf if the surface field is $2 \times$ $10^{7}$ gauss. Emission takes place in a range of cyclotron harmonics centered about harmonic 40 , and the average energy of the electrons is roughly $350 \mathrm{keV}$. The origin of the electrons is unknown.

The accretion of matter onto white dwarf stars has been studied by many authors, and references may be found in [60]. A standing shock wave is formed above the white dwarf surface. Hard $X$-rays are emitted by bremsstrahlung from infalling matter in the hot post-shock region; soft $X$-rays may be emitted thermally from the white dwarf surface by absorption and re-emission. In many cataclysmic variables the white dwarf is a member of a binary system, and the accretion will produce an accretion disk. A strong magnetic field modifies the accretion by channeling the matter towards one or both magnetic poles once it has crossed the Alfven surface (where the kinetic energy density of the infalling matter is equal to the magnetic energy density). It seems 1ikely [63] that at the inner Lagrangian point of AM Herculis the magnetic energy density would indeed exceed the kinetic energy density, so that there will be no accretion disk [68]. The Alfven surface will enclose the companton M star. Cyclotron emission may be an important mechanism, and the emission may take place in higher harmonics [69] because the lowest harmonics are self-absorbed. Mode1s of AM Herculis have been constructed. The earliest models $[56,57]$ assumed an accretion disk. Later mode1s $[68,70,71]$ assumed magnetic funneling of the accreting material, and the magnetic axis is inclined to the rotation axis. There are still features which are unexplained by the models so far constructed, and further investigations will be needed. 
AN Ursae Majoris. This is a magnetic white dwarf in a binary system, similar to AM Herculis. Circular polarization varying between $-9 \%$ and $-35 \%$ was found by Krzeminski and Serkowski [72]. The period is 1.91 hours. The inferred magnetic field of the white dwarf is $3 \times 10^{8}$ gauss. $X$-ray emission has been detected by Hearn and Marshal1 [73]. Five color photometry was reported by Gilmozzi, Messi and Natali [74].

EF Eridani $=2 \mathrm{~A}$ 0311-227. This binary has a period of 81 minutes. It was identified by Griffiths et al. [75]. Polarization was detected by Tapia [76], photometry was performed by Bond, Chanmugam and Grauer [77], spectroscopy by Crampton, Hutchings and Cowley [78] and $X$-ray measurements by White [79]. There is evidence [78] that the magnetic field might have a complex structure, perhaps quadrupolar. An absorption dip in the X-ray spectrum [79] is thought to be associated with the accretion column in front of the $\mathrm{X}$-ray source.

VV Puppis. This nova-1ike variable was discovered by Herbig [80] to be a binary system. Its period is 100 minutes. Its light curve is like an eclipsing variable except that the minimum lasts more than half the period. It has variable linear and circular polarization, discovered by Tapia [81], it must have a substantial region of $11 \mathrm{ght}$ emission where the fleld exceeds $10^{8}$ gauss [10], and 1 ts companion star $1 \mathrm{~s}$ probably a falnt red dwarf with absolute magnitude fainter than 15 [82]. The opt1cal spectrum, observed by Wickramasinghe and V1svanathan [83] shows features believed to be the only known case of cyclotron emission seen as such. Confirmation of the features was obtained by Stockman, Liebert and Bond [84]. The even spacing of several absorption features strongly supports the cyclotron theory of their emission. The spacing corresponds to a field of about $2.7 \times 10^{7}$ gauss. The absorption appeared whenever the primary emission region was in view. Models [84,85] assume that the emission arises from a region small compared with the size of the white dwarf, situated near one of the magnetic poles, and eclipsed by the white dwarf as it rotates synchronously with the orbital motion. The optical luminosity can be explained in terms of accretion by the white dwarf.

DQ Herculis. Th1s star is the remnant of Nova Herculis 1934 . There is no evidence for magnetic fields as yet, but a magnetic field might be responsible for the observed speeding up of the rotation. The situation was reviewed by Angel [10].

4. The Crab Pulsar. A number of measurements have been made of the $X$-ray spectrum of the Crab nebula and its pulsar. Ling, Mahoney, Willett and Jacobson [86] reported evidence of a narrow line at $73 \mathrm{keV}$ with a time-averaged intensity of $3.8 \times$ $10^{-3}$ photons $\mathrm{cm}^{-2} \mathrm{sec}^{-1}$. Strickman, Johnson and Kurfess [87] observed the 20$250 \mathrm{keV}$ range and analyzed their results by assuming that all the unpulsed emission comes from the nebula and the pulsed emission from the pulsar. They did not detect a line at $73 \mathrm{keV}$, and gave upper limits of $1.3 \times 10^{-3}$ photons $\mathrm{cm}^{-2} \mathrm{sec}^{-1}$ to a line from the nebula and $4.9 \times 10^{-4}$ photons $\mathrm{cm}^{-2} \mathrm{sec}^{-1}$ to a Iine from the pulsar. other observers have claimed to detect or not to detect the line. If the line is real it must be variable.

Strickman, Kurfess and Johnson [88] have re-analyzed their earlier observations as a function of position in the pulse cycle as well as a function of energy. They detected a line at $77 \mathrm{keV}$ wth an intensity $4.1 \times 10^{-3}$ photons $\mathrm{cm}^{-2} \mathrm{sec}^{-1}$ and a line width wholly instrumental. The feature appears only in the 70-89 keV energy bin, and not in other energy ranges, and only during pulse periods, so that it is pulsed at the pulse frequency. A definitive detection was also reported by Manchanda, Bazzano, La Padula, Polcaro and Ubertini [89]. Their 1ine intensity is $5 \times 10^{-3}$ photons $\mathrm{cm}^{-2} \sec ^{-1}$.

Cyclotron emission seems the likely mechanism for producing the line. If the energy of $77 \mathrm{keV}$ corresponds to the cyclotron frequency a magnetic field of $6 \times 10^{12}$ gauss is indicated. Correcting for a gravitational red shift would increase this value somewhat. If the electrons are streaming out from the pulsar with relativistic velocities the magnetic field required could be as low as $10^{10}$ gauss, but in any case 
this is still quite close to the pulsar. There are many problems associated with the proposed cyclotron mechanism. The observed narrow line requires a large number of electrons to have similar behavior (same Lorentz factors). An energy injection mechanism is required to make up for the cyclotron radiative losses. The magnetic field in the emfssion region must be nearly constant, implying a small volume of space. Clearly we have much to learn about the Crab pulsar.

Acknowledgment. Preparation of this paper was supported in part by National Science Foundation Grant PHY79-04928 to the University of Colorado.

\section{References}

[1] BABCOCK H. W., Astrophys. J. I05 (1947) 105-119.

[2] KEMP J. C., SWEDLUND J. B., LANDSTREET J. D. and ANGEL J. R. P., Astrophys. J. (Letters) 161 (1970) L77-79.

[3] GREENSTEIN J. L., Astron. J. 81 (1976) 323-338.

[4] LIEBERT J., Ann. Rev. Astron. Astrophys. 18 (1980) 363-398.

[5] PRESTON G W., Astrophys. J. (Letters) 160 (1970) L143-145.

[6] ELIAS J. H. and GREENSTEIN J. L., Pub1. Astron. Soc. Pacific 86 (1974) 957959.

[7] ANGEL J. R. P. and LANDSTREET J. D., Astrophys. J. (Letters) 160 (1970) L147152.

[8] ANGEL J. R. P., BORRA E. F. and LANDSTREET J. D., Astrophys. J. SuppI. 45 (1981) 457-474.

[9] GaRstang R. H., Rep. Prog. Phys. 40 (1977) 105-154.

[10] ANGEL J. R. P., Ann. Rev. Astron, Astrophys. 16 (1978) 487-519.

[11] ANGEL J. R. P., CARSWELL R. F., STRITTMATTER P. A., BEAVER E. A. and HARMS R., Astrophys. J. (Letters) 194 (1974) L47-50.

[12] GREENSTEIN J. L., Astrophys. J. (Letters) 194 (1974) L51-55.

[13] KEMIC S. B., Joint Institute for Laboratory Astrophysics, Boulder, Colorado (1974) Report No. 113, 1-27.

[14] BROWN D. N., RICH A., WILLIAMS W. L. and VAUCLAIR G., Astrophys. J. 218 (1977) 227-231.

[15] WICKRAMASINGHE D. T. and BESSEL M. S., Astrophys. J. (Letters) 203 (1976) L3941.

[16] WICKRAMASINGHE D. T., WHELAN J. A. J. and BESSEL M. S., Mon. Not. Roy. Astron. Soc. 180 (1977) 373-378.

[17] MARTIN B. and WICKRAMASINGHE D. T., Mon. Not. Roy. Astron. Soc. 183 (1978) 533-538.

[18] LIEBERT J., ANGEL J. R. P., STOCKMAN H. S., SPINRAD H. and BEAVER E. A., Astrophys. J. 214 (1977) 457-470.

[19] GREENSTEIN J. L. and BOKSENBERG A., Mon. Not. Roy. Astron. Soc. 185 (1978) 823-832.

[20] GREENSTEIN J. L., Pub. Astron. Soc. Pacific 90 (1978) 303-306.

[21] GREENSTEIN J. L., GUNN J. E. and KRISTIAN J., Astrophys. J. (Letters) 169 (1971) L63-69.

[22] WEGNER G., Mon. Not. Roy. Astron. Soc. 174 (1976) 191-202.

[23] LIEBERT J., ANGEL J. R. P. and LANDSTREET J. D., Astrophys. J. (Letters) 202 (1975) $1139-143$.

[24] ANGEL J. R. P. and LANDSTREET J. D., Astrophys. J. (Letters) 178 (1972) L2122.

[25] LANDSTREET J. D. and ANGEL J. R. P., Astrophys. J. (Letters) 165 (1971) L6770 .

[26] GREENSTEIN J. L., Astrophys. J. 158 (169) 281-293.

[27] GREENSTEIN J. L., Astrophys. J. (Letters) 162 (1970) L55-59.

[28] ANGEL J. R. P. and LANDSTREET J. D., Astrophys. J. 191 (1974) 457-464.

[29] LIEBERT J., ANGEL J. R. P., STOCKMAN H. S. and BEAVER E. A., Astrophys. J. 225 (1978) 181-190.

[30] ANGEL J. R. P. and LANDSTREET J. D., Astrophys. J. (Letters) 162 (1970) L6166. 
[31] KEMP J. C. and SWEDLUND J. B., Astrophys. J. (Letters) 162 (1970) L67-68.

[32] ANGER J. R. P., LANDSTREET J. D. and OKE J. B., Astrophys. J. (Letters) 171 (1972) L11 15.

[33] LANDSTREET J. D. and ANGEL J. R. P., Astrophys. J. 196 (1975) 819-825.

[34] MINKOWSKI R., Carnegie Institute of Washington Yearbook No. 37 (1938) 200.

[35] GREENSTEIN J. L., in Proc. 3rd Symp. Math. Statistics and Probability, Vo1. 3 (ed. J. Neyman, Univ, California Press, 1956), 11-30.

[36] GREENSTEIN J. L. and MATTHEWS M. S., Astrophys. J. 126 (1957) 14-18.

[37] WEGNER G., Pub. Astron. Soc. Pacific 83 (1971) 205-206.

[38] ANGEL J. R. P., in White Dwarfs and Varlable Degenerate Stars, Int. Astron. Union Colloquium No. 53 (eds. H. M. Van Horn and V. Weidemann, Univ. Rochester, 1979), 313-316.

[39] SWEDLUND J. B., WOLSTENCROFT R. D., MICHALSKY J. J. and KEMP J. C., Astrophys . J. (Letters) 187 (1974) L121-123.

[40] KEMP J. C., COYNE G. V., SWEDLUND J. B. and WOLSTENCROFT R. D., Astrophys. J. (Letters) 189 (1974) L79-80.

[41] LANDSTREET J. D. and ANGEL J. R. P., Astrophys. J. (Letters) 190 (1974) L2526.

[42 J GREENSTEIN J. L., SCHMIDT M. and SEARLE L., Astrophys. J. (Letters) 190 (1974) L27-28.

[43] LIEBERT J., Pub. Astron. Soc. Pacific 88 (1976) 490-494.

[44] GREEN R. F. and LIEBERT J., Pub. Astron. Soc. Pactfic 93 (1981) 105-109.

[45] LIEBERT J. and STOCKMAN H. S., Pub. Astron. Soc. Pacific 92 (1980) 657-660.

[46] ANGEL J. R. P. and LANDSTREET J. D., Astrophys. J, (Letters) 164 (1971) L1516.

[47] KEMP J. C., SWEDLUND J. B. and WOLSTENCROFT R. D., Astrophys. J. (Letters) 164 (1971) L17-18.

[48] ANGEL J. R. P., ILLING R, M. E. and LANDSTREET J. D., Astrophys. J. (Letters) 175 (1972) L85-87.

[49] ANGEL J. R. P. and LANDSTREET J. D., Astrophys. J. (Letters) 165 (1971) L7175.

[50] ANGEL J. R. P., HINTZEN P. and LANDSTREet J. D., Astrophys. J. (Letters) 196 (1975) L.27-29.

[51] ANGEL J. R. P., HINTZEN P., STRITTMATTER P. A. and MARTIN P. G., Astrophys. J. (Letters) 190 (1974) L7 1-72.

[52] MARTIN B. and WICKRAMAS INGHE D. T., Mon. Not. Roy. Astron. Soc. 189 (1979) 6977.

[53] GREENSTEIN J. L. and OKE J. B., Astrophys. J. 252 (1982) 285-295.

[54] BERG R. A. and DUTHIE J. G., Astrophys. J. 211 (1977) 859-865.

[55] HEARN D. R. and RICHARDSON J. A., Astrophys. J. (Letters) 213 (1977) L115-117.

[56] SZKODY P. and BROWNLEE D. E., Astrophys. J. (Letters) 212 (1977) L113-116.

[57] COWLEY A. P. and CRAMPTON D., Astrophys. J. (Letters) 212 (1977) L121-124.

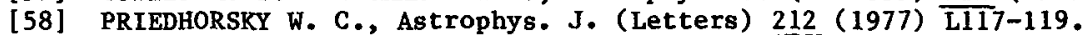

[59] TAPIA S., Astrophys. J. (Letters) 212 (1977) L1 25-129.

[60] CHIAPPETTI L., TANZI E. G. and TREVES A., Space Sci. Rev. 27 (1980) 3-33.

[61] CHANMUGaM G. and DULK G. A., Astrophys. J. (Letters) 255 (1982) L107-110.

[62] SCHMIDT G. D., STOCKMAN H. S. and MARGON B., Astrophys. J. (Letters) 243 (1981) L157-161 .

[63] YOUNG P., SCHNELDER D. P. and SHECTMAN S. A., Astrophys. J. 245 (1981) 10431053.

[64] LATHAM D. W., LIEBERT J. and STEINER J. E., Astrophys. J. 246 (1981) 919-934.

[65] HUTCHINGS J. B., CRAMPTON D. and COWLEY, A., Astrophys. J. 247 (1981) 195-201.

[66] ROTHSCHILD R. E., GRUBER D. E., KNIGHT F. K., MATTESON J. L., NOLAN P. L., SWANK J. H., HOLT S. S., SERLEMITSOS P. J., MASON K. O. and TUOHY I. R,, Astrophys. J. 250 (1981) 723-732.

[67] TUOHY I. R., MASON K. 0., GARMIRE G. P. and LAMB F. K., Astrophys. J. 245 (1981) 183-194.

[68] CHANMUGAM G. and WAGNER R. L., Astrophys. J. (Letters) 213 (1977) 1.13-16.

[69] CHANMUGAM G. and WAGNER R. L., Astrophys. J. 232 (1979) 895-902.

[70] CHANMUGAM G. and WAGNER R. L., Astrophys. J. $\frac{222}{22}$ (1978) 641-646.

[71] PRIEDHORSKY W. C. and KREZEMINSKI, W., Astrophys, J. 219 (1978) 597-604.

[72] KRZEMINSKI W. and SERKOWSKI K., Astrophys. J. (Letters) 216 (1977) L45-48. 
[73] HEARN D. R. and MARSHALL F. J., Astrophys. J. (Letters) 232 (1979) I.21-23.

[74] GILMOZZI R., MESSI R. and NATALI G., Astrophys. J. (Letters) 245 (1981) L119122.

[75] GRIFFITHS R. E., WARD M. J., BLADES J. C., WILSON A. S., CHAISSON L. and JOHNSTON M. D., Astrophys. J. (Letters) 232 (1979) L.27-31.

[76] TAPIA S., Internat. Astron. Union Circ. $\overline{3327}$ (1979).

[77] BOND H. E., CHANMUGAM G. and GRAUER A. D., Astrophys. J. (Letters) 234 (1979) L113-116.

[78] CRAMPTON D., HUTCHINGS J. B. and COWLEY A. P., Astrophys. J. 243 (1981) 567575 .

[79] WHITE N. E., Astrophys. J. (Letters) 244 (1981) L85-88.

[80] HERBIG G. H., Astrophys. J. 132 (1960) 76-86.

[81] TAPIA S., Internat. Astron. Union Circ. 3054 (1977).

[82] LIEBERT J., STOCKMAN H. S., ANGEL J. R. P., WOLFF N. J., HEGE K. and MARGON B., Astrophys. J. 225 (1978) 201-208.

[83] WICKRAMASINGHE D. T. and VISVANATHAN N. V., Mon. Not. Roy. Astron. Soc. 191 (1980) 589-598.

[84] STOCKMAN H. S., LIEBERT J. and BOND H. E., White Dwarfs and Variable Degenerate Stars, Internat. Astron. Union Colloq. No. 53 (eds. H. M. Van Horn and V. Wiedemann, Univ. Rochester, 1979).

[85] WICKRAMASINGHE D. T. and MEGGITT S. M. A., Mon. Not. Roy. Astron. Soc. 198 (1982) 975-983.

[86] LING J. C., MAHONEY W. A., WILlETT J. B. and JACOBSON A. S., Astrophys. J. 231 (1979) 896-905.

[87] STRICKMAN M. S., JOHNSON W. N. and KURFESS J. D., Astrophys. J. (Letters) 230 (1979) L15-19.

[88] STRICKMAN M. S., KURFESS J. D. and JOHNSON W. N., Astrophys. J. (Letters) 253 (1982) L23-27.

[89] MANCHANDA R. K., BAZZANO A., LA PADULA C. D., POLCARO V. F. and UBERTINI P., Astrophys . J. 252 (1982) 172-178. 